\title{
Current Treatment Options in Acute Limb Ischemia
}

\section{Aktuelle Behandlungsmöglichkeiten der akuten Extremitätenischämie}

Authors

Friederika Fluck, Anne Marie Augustin, Thorsten Bley, Ralph Kickuth

\author{
Affiliation \\ Institute of Diagnostic and Interventional Radiology, \\ University-Hospital of Würzburg, Würzburg, Germany \\ Key words \\ ischemia/infarction, thrombolysis, embolism/thrombosis, \\ extremities, interventional procedures, acute
}

received 29.12 .2018

accepted 04.08.2019

Bibliography

DOI https://doi.org/10.1055/a-0998-4204

Published online: 28.8.2019

Fortschr Röntgenstr 2020; 192: 319-326

(c) Georg Thieme Verlag KG, Stuttgart · New York

ISSN 1438-9029

Correspondence

Dr. Friederika Fluck

Department of Diagnostic and Interventional Radiology, Universitätsklinikum Würzburg, Oberduerrbacher Str. 6, 97080 Würzburg, Germany

Tel.: ++49/931/20134001

Fax: ++49/931/201634001

fluck_f@ukw.de

\section{ZUSAMMENFASSUNG}

Hintergrund Die akute Ischämie der unteren Extremität stellt einen klinischen Notfall mit möglichem Extremitätenverlust und lebensgefährlichen Folgen dar. Sie ist durch eine plötzliche Minderdurchblutung der Extremität gekennzeichnet. Definitionsgemäß weist die akute Ischämie eine Symptomdauer bis zu 14 Tagen auf. Die Prävalenz der akuten Extremitätenischämie ist durch die zunehmende Lebenserwartung in den letzten Jahren gestiegen. Die Thrombembolie und Lokalthrombose sind die häufigsten ätiologischen Faktoren. Ausschlaggebend für den Extremitätenerhalt ist die sofortige Diagnosestellung und Initiierung des therapeutischen Prozederes. Eine Verzögerung von Diagnose und Therapie kann zu irreversiblen ischämischen Schäden führen.

Methoden Diese Übersichtsarbeit basiert auf einer selektiven Literaturrecherche in PubMed, die den aktuellen Forschungsstand repräsentiert.

Ergebnisse und Schlussfolgerungen Patienten mit akuter Ischämie der unteren Extremität sollten umgehend antikoa- guliert werden. Das weitere diagnostische und therapeutische Prozedere ist abhängig vom Schweregrad der Ischämie. Insbesondere die akuten (<14 Tage Symptomdauer) Rutherford-Kategorien Ila und Ilb mit marginaler und unmittelbarer Gefährdung der Extremitäten erfordern eine definitive therapeutische Intervention und sind im Falle einer prompten Revaskularisation beinerhaltend angehbar. Bislang galt die offen chirurgische Revaskularisation als zeiteffektiver im Vergleich zur lokalen Thrombolyse. Durch die Entwicklung neuer perkutaner mechanischer Katheterthrombektomie-Systeme ist die Behandlungszeit verkürzt und erfolgsversprechende Ergebnisse bezüglich Extremitätenerhalt und Überlebensraten wurden berichtet. Es fehlen randomisierte Studien und die Leitlinien empfehlen, die Methode der schnellstmöglichen Revaskularisation in Abhängigkeit von Lokalisation, Ätiologie und persönlicher Expertise zu wählen.

\section{Kernaussagen:}

- Die akute Ischämie der unteren Extremität ist ein interdisziplinärer Notfall. Er kann zum Extremitätenverlust führen und lebensbedrohliche Folgen haben.

- Sofortige Diagnosestellung und Therapieeinleitung sind entscheidend, um irreversible Schäden zu vermeiden.

- Der endovaskuläre Behandlungsansatz sollte in den Kategorien Ila und IIb nach der Rutherford-Klassifikation für akute Extremitätenischämie (<14 Tage) erfolgen. Insbesondere ältere, multimorbide Patienten mit hohem perioperativem Risiko profitieren (mutmaßlich) von der minimalen Invasivität.

\section{ABSTRACT}

Background Acute limb ischemia represents a clinical emergency with eventual limb loss and life-threatening consequences. It is characterized by a sudden decrease in limb perfusion. Acute ischemia is defined as a duration of symptoms for less than 14 days. Aging of the population increases the prevalence of acute limb ischemia. The two principal etiologies are arterial embolism and in situ thrombosis of an atherosclerotic artery. Immediate diagnosis, accurate assessment and urgent intervention when needed are crucial to save the limb and to prevent a major amputation. Delay in diagnosis and therapy may lead to irreversible ischemic damage.

Method To assess the current treatment options in acute limb ischemia, this review is based on a selective literature search in PubMed representing the current state of research. 
Results and Conclusion Patients with acute limb ischemia should receive immediate anticoagulation. Treatment depends on the classification based on the degree of ischemia and limb viability. Especially acute ( $<14$ days symptom duration) Rutherford Categories Ila and IIb with marginally and immediately threatened limbs require definitive therapeutic intervention and are salvageable, if promptly revascularized. The current literature suggests that open surgical revascularization is more time effective then catheter-directed thrombolysis. However, with the advent of thrombolytic delivery systems and mechanical thrombectomy devices, treatment time can be minimized and successful utilization in patients with Category IIb (Rutherford Classification for Acute Limb Ischemia) has been reported with promising limb-salvage and survival rates. Large randomized studies are still missing, and guidelines suggest choosing the method of revascularization depending on anatomic location, etiology, and local practice patterns, with the time to restore the blood flow being an important factor to consider.

\section{Key points:}

- Acute limb ischemia is an interdisciplinary emergency. It can lead to limb loss with life-threatening consequences.

- Immediate diagnosis and treatment are crucial to prevent irreversible damage.

- An endovascular approach should be considered in acute limb ischemia Category Ila and Ilb of the Rutherford Classification for Acute Limb Ischemia (<14 days), on a case-based decision and local capabilities. Especially elderly, multimorbid patients with high perioperative risk (may probably) benefit from these minimally invasive procedures.

\section{Citation Format}

- Fluck F, Augustin A, Bley T et al. Current Treatment Options in Acute Limb Ischemia. Fortschr Röntgenstr 2020; 192: 319-326

\section{Background}

Acute limb ischemia (ALI) is a sudden, recent (less than 14 days duration) decrease in limb perfusion resulting in a threat to the viability of the lower extremity [1]. It is one of the most frequent causes for major amputation affecting approximately 1.5 persons per 10000 per year [2]. Acute renal insufficiency, hyperkalemia and metabolic acidosis are possible systemic consequences of untreated ALI and are often life-threatening. Major amputation after ALI ranges between $10 \%$ and $15 \%$ while the associated 30 -day mortality is documented as 15-25\% [3]. The etiology of non-traumatic ALI can be categorized as embolic (30\%), thrombotic (60\%), or thrombosis of an existing stent or bypass graft. Cardiac emboli are the most common source of embolism (75\%), with atrial fibrillation, recent myocardial infarction with mural thrombi, endocarditic vegetations and atrial myxoma as possible underlying causes. Aneurysmal diseased segments and proximal atherosclerotic debris are another source of emboli, when debris originating in proximal vessels dislodges and obstructs peripheral arteries. Less frequently, embolism is due to air, fat, amniotic fluid, or systemic tumor fragments or is iatrogenic. In rare cases paradoxical venous embolism in patients with a patent foramen ovale have been reported. Embolism recurs in 6-45\%. Therefore, anticoagulation is important to prevent recurrence [4]. Anticoagulation leads to a reduction in the propagation of the thrombus and accumulation of free-floating thrombus formations. In general, indirect anticoagulants such as heparin, Vitamin K antagonists like phenprocoumon or warfarin need to be differentiated from direct anticoagulants like thrombin or factor Xa inhibitors. While direct oral anticoagulants (Non-Vitamin K Antagonist Oral Anticoagulant $=$ NOACs) bind to and inhibit the activity of thrombin, indirect anticoagulants require a cofactor to interact with thrombin and therefore prevent blood clot formation. Heparin as an indirect inhibitor is administered sc. or iv., shows immediate effect, and is used in the initial management of ALI. On the other hand, thrombocyte aggregation inhibitors prevent platelets from clumping and are recommended in patients with underlying peripheral arterial disease, to reduce the risk of myocardial infarction, stroke, or chronic critical ischemia [5]. In addition, platelet inhibitors are used during and after peripheral arterial interventions, especially PTA with or without stent implantation.

In situ thrombosis occurs in a diseased local arterial segment with prior gradual atherosclerotic narrowing stimulating the formation of collateral channels. Rupture of an atherosclerotic plaque resulting in thrombus formation and arterial occlusion is discussed as the underlying pathomechanism [3].

Once the diagnosis is suspected, the severity of limb ischemia should be determined using the currently accepted classification system, introduced by the Society for Vascular Surgery (SVS) and adapted from the Rutherford classification (TASC II). In 1986, Rutherford first categorized acute and chronic limb ischemia based on clinical symptoms with objective (noninvasive) findings, emphasizing that the timing of symptom onset and different treatment algorithms are required. Unlike acute limb ischemia (ALI), critical limb ischemia (CLI) is defined by chronic ischemic rest pain, nocturnal recumbent pain, ischemic skin lesions, and symptom duration for at least 2 weeks [6]. To ensure clarity in communication and influence the urgency of revascularization, further therapeutic intervention is based on this clinical classification $[7,8]$ ( $\triangleright$ Table 1$)$.

Patients presenting with ALI have become progressively older. They suffer from multiple medical comorbidities that elevate the perioperative risk of surgical interventions. Therefore, endovascular strategies appear to be beneficial, especially in those patients.

Retrospective studies have investigated the efficacy and safety of endovascular therapies for ALI, with some suggesting improved limb salvage and mortality [9, 10]. In the most recent analysis, endovascular approaches aside from catheter-directed thrombolysis 
- Table 1 Classification of Acute Limb Ischemia (TASC II) (adapted from the Rutherford classification [5] by SVS [1]).

\begin{tabular}{|l|l|l|l|l|}
\hline category & prognosis & sensory loss & motor impairment & arterial Doppler \\
\hline $\begin{array}{l}\text { I } \\
\text { viable }\end{array}$ & no immediate limb threat & no & no & audible \\
\hline $\begin{array}{l}\text { Ila } \\
\text { threatened: marginal }\end{array}$ & salvageable if treated promptly & $\begin{array}{l}\text { none or minimal } \\
\text { (toes) }\end{array}$ & no & often inaudible \\
\hline $\begin{array}{l}\text { IIb } \\
\text { threatened: immediate }\end{array}$ & $\begin{array}{l}\text { salvageable if treated } \\
\text { immediately }\end{array}$ & $\begin{array}{l}\text { more than toes } \pm \\
\text { rest pain }\end{array}$ & mild to moderate & usually inaudible \\
\hline $\begin{array}{l}\text { III } \\
\text { irreversible }\end{array}$ & permanent damage & profound & profound & audible \\
\hline
\end{tabular}

seem also to be cost-effective when compared with surgical intervention, since they are associated with a shorter in-hospital stay and there is no need for beds in an intensive care unit [11]. Endovascular intervention and surgical treatment should be viewed as complementary rather than competing approaches for ALI.

\section{Complementary imaging}

Since ALI is a clinical diagnosis, further investigations are carried out to confirm etiology and level/location of occlusion and to plan the therapeutic approach (e. g. access side). Based on fast availability, noninvasive imaging methods can be considered equal in patients categorized as IIb according to the Rutherford classification for acute limb ischemia allowing for better therapy planning [12].

Conventional arteriography (digital subtraction angiographyDSA), the former gold standard, is progressively being replaced by less invasive modalities [13]. It offers interventional therapeutic options for ALI but carries the risk of procedure-related complications (such as access bleeding), ionizing radiation and exposure to iodinated contrast medium. When renal function is normal, there seems to be no risk of contrast-induced acute kidney injury [14]. However, in patients with known impaired renal function, the risk for contrast-induced acute kidney injury may increase with eGFR $<30 \mathrm{~mL} / \mathrm{min} / 1.73 \mathrm{~m}^{2}$ (ESUR Guidelines 10.0).

MR angiography has undergone significant advances in the past years providing good quality images with high sensitivity and specificity, compared to DSA. With the advent of numerous technical innovations, decreased total examination time coupled with a simultaneously clearer depiction of small vessels can be achieved [15]. Limitations are the need for more time-consuming sequences to get a better resolution of smaller arteries and reported overestimation of stenosis in native arteries and secondary to artifacts in case of vascular stents [16]. The risk of acute kidney injury is very low when gadolinium-based contrast agents are used in approved doses (ESUR Guidelines 10.0). Compared to conventional or CT angiography, it is rather time-consuming and often not feasible or available in the emergency setting.

$\mathrm{CT}$ angiography is noninvasive, readily available, cost-effective and can be used in patients with prior stents, pacemaker, or defibrillators (unlike MRA). It has proven to have comparable accuracy to MRA and has excellent spatial resolution. Advantages compared to conventional arteriography include multiplanar visualization and reconstruction, which can be useful for the evaluation of stenosis and identification of collateral vessels. In heavily calcified vessels, the ability to depict the lumen is limited despite editing algorithms, and dual-energy CTA is still said to overestimate high-grade vessels stenosis [17].

Duplex ultrasound enables identification and location determination and establishes the patency of outflow vessels. The main limitations are the inability to get a complete radiological road map, the fact that the method is operator-dependent, and technical limitations with respect to assessing the iliac arteries. The sensitivity for ultrasound decreases when the occlusion is located at or below calf level and in the case of heavy calcifications, and poor overall accuracy is known if multilevel disease is present [13].

To sum up, the preferred noninvasive imaging modality is commonly CT angiography, as it may promptly identify the level of the occlusion as well as the inflow and outflow situation, may demonstrate prior surgical or endovascular intervention, and may be obtained quickly. Nevertheless, the choice of diagnostic approach depends on the general patient condition and personal and technical conditions of each department.

\section{Management of ALI}

The first step in initial ALI management is immediate anticoagulation with heparin [8]. It was first introduced in 1978 by Blaisdell et al. [18] to prevent proximal and distal propagation of the thrombus. Heparin has no effect on the lysing of the established thrombus. It prevents thrombus formation and thereby avoids secondary thrombosis proximal and distal to the lesion and preserves the microcirculation. Patients should receive appropriate analgesia and proper handling of the limb and hydration, which may attenuate the contrast load they will receive and the potential myoglobinuria from reperfusion.

A treatment selection factor that is decisive for the treatment of $\mathrm{ALI}$ is the Rutherford classification based on the degree of ischemia and limb viability. Other factors guiding further clinical management are the duration of symptoms, surgical risk factors, 
contraindications for thrombolysis, anatomical location, etiology and overall patient condition [8].

Category I ALI patients presenting with a viable limb may benefit from noninvasive diagnostic imaging and noninvasive vascular and laboratory studies to ascertain possible underlying chronic vascular disease. The need for elective vascular intervention can be determined while optimizing risk factors $[8,19]$.

Category II ALI patients require urgent or emergent revascularization to prevent progression to category III. The duration of symptoms, contraindications, etiology and location of the occlusion are decisive for therapeutic options in category lla ALI. Endovascular therapy proved to be superior to surgery to treat acute category lla events when the duration of symptoms is less than 14 days $[20,21]$. Patients who present contraindications or have an incomplete response to thrombolysis or symptoms lasting longer than 14 days may benefit from surgical revascularization. In general, embolic disease should be considered if the occlusion lies at a vessel bifurcation or trifurcation [12].

Category IIb ALI indicates a profoundly ischemic limb, and immediate revascularization is indicated [1]. Due to the associated time for reestablishing vascularization with catheter-directed thrombolysis (CDT), traditionally open surgical revascularization has been performed. However, with the advent of mechanical thrombectomy devices, the treatment time can be minimized and successful utilization in patients with category IIb ALI is reported with increasing frequency [8]. More recent case series reported similar revascularization rates with endovascular treatment with decreased 30-day morbidity and mortality compared to open surgery $[12,19]$.

Large multicenter trial data for this group of patients are still missing and the guidelines suggest choosing the method of revascularization depending on the anatomic location of the occlusion, etiology of ALI, contraindications to open or endovascular treatment and local practice patterns [1, 22].

Category III ALI patients present major tissue loss along with permanent nerve damage. Revascularization is not indicated and can actually lead to reperfusion-related organ dysfunction and death. After initial stabilization, amputation should be considered.

In general, patients with category I ALI need urgent revascularization (e.g. within 12 hours of presentation), while patients with category II ALI need emergent revascularization (e.g. within 26 hours of presentation) [12].

Patients presenting with an acute graft occlusion usually suffer from progression of their atherosclerosis as the underlying causative reason. The guidelines suggest thrombolysis with subsequent PTA/stenting or surgical revision [1]. Local lysis is associated with bleeding complications and intensive patient monitoring. Surgical thrombectomy on the other hand shows perioperative complications and only small technical success rates in randomized trials $[3,23,24]$. Percutaneous mechanical thrombectomy (PMT) has also been proven to be an effective and safe therapy option in addition to the established lysis therapy [25].

\section{Endovascular treatment technique}

Four consecutive steps are necessary for an endovascular approach: Diagnostic angiography, lesion crossing, management of the thrombus, and an approach with special regard to a potentially underlying lesion. Reperfusion of the affected limb can be achieved by use of a thrombolytic agent alone or in conjunction with a mechanical device for aspiration, fragmentation of the thrombus, angioplasty, and optional stenting. Bleeding, vascular injury, and distal embolization are some of the associated complications [19]. There is a lack of prospective data. However, the use of adjunctive mechanical therapies has increased over time, supported by observational analysis [26].

The choice will depend on the presence of a neurological deficit, duration of symptoms, affected artery, patients' comorbidities and risk factors, whether it is a native artery, therapy-related risks and outcomes. In more severe cases with neurological deficit, extraction and aspiration of the thrombus or surgical therapy are preferable. In patients with threatened limbs, the time to reestablish perfusion by thrombolysis is usually regarded as too long and the development of compartment syndrome is reported in up to $10 \%$ of cases [27]. In the absence of neurological deficit, catheter-directed thrombolytic therapy is more appropriate [28].

\section{Catheter-Directed Thrombolysis (CDT)}

The CDT approach is recommended in Category-Ila ALI with a short duration of symptomatology (<14 days) where the thrombus is still fresh and there is adequate time for the thrombolytic agent to perform its intended function. In the literature, information about dosing and duration of thrombolytic therapy differs extensively and can range between 2.5 and 48 hours in the exemplary case of alteplase $[12,29]$. The following protocols are recommended: 1 . weight-adapted administration of alteplase with $0.001-0.02 \mathrm{mg} / \mathrm{kg} / \mathrm{h}$ or 2 . Non-weight-based administration of alteplase with $0.12-2.0 \mathrm{mg} / \mathrm{h}$ (maximum dose: $40 \mathrm{mg}$ ). In both scenarios additional low-dose intraarterial heparin (400-600 IU/h) to avoid new thrombus formation should be discussed [20]. A more recent study found that the addition of continuous heparin infusion during intra-arterial thrombolysis had no significant benefit and was associated with a few major bleeding complications (30.3\%) [30]. Although most of the reported bleeding episodes were mild and could be managed without surgical intervention, especially in elderly multimorbid patients, the associated risks must be weighed individually. For reteplase subsequent protocols are recommended: From 0.25 to $1.0 \mathrm{mg} / \mathrm{h}$ (maximum dose: $20 \mathrm{IU}$ in $24 \mathrm{~h}$ ) or in a low-dose regimen: $0.125 \mathrm{mg} / \mathrm{h}$. Tenecteplase should be administrated as a bolus infusion of $1-5 \mathrm{mg}$, followed by infusions ranging from $0.125-0.5 \mathrm{mg} / \mathrm{h}$ [20].

An increased risk of hemorrhagic complications is said to occur in low-dose, long-duration thrombolytic infusions. On the other hand, an elevated risk of distal embolization may be associated with high-dose, short-duration thrombolytic infusions. The infusion of the thrombolytic agent may be continuous, pulse-spray, or initial pulse-spray with subsequent continuous infusion, the idea behind the latter being to more rapidly penetrate and induce 
fragmentation of the thrombus $[12,29]$. To date, there is no standardization of the therapeutic protocol in regard to dosing and duration of the thrombolytic agent or monitoring of the patient. When comparing standard thrombolysis with ultrasound-accelerated thrombolysis (UST), a multicenter Dutch trial showed a significantly reduced therapy time with UST, but with a substantial number of bleeding complications [31]. Large cohort studies or systematic meta-analyses to show clinical benefits are missing [31-33].

There are different generations of thrombolytic agents, with each generation achieving increased specificity for fibrin-bound plasminogen. First-generation thrombolytics (streptokinase, urokinase) are no longer used due to the increased risk of hemorrhagic complications; second-generation agents (alteplase) act practically solely on fibrin-bound plasminogen; third-generation agents have less affinity for free circulating plasminogen (reteplase) or better resistance to endogenous inactivating factors in the plasma (tenecteplase) [12, 29]. Alfimeprase, a novel recombinant variant of fibrolase (direct fibrinolytic enzyme with no effect of plasminogen), first showed promising results in preclinical and pilot studies with regard to duration of therapy and risk of bleeding. In a multicenter study with two blinded, placebo-controlled, randomized trials, alfimeprase did not show life-threatening bleeding complications, but did not have greater effectiveness than placebo either [34].

Currently, in most cases, CDT is combined with target lesion endovascular/surgical correction to improve long-term patency rates and to decrease the amputation rate [35].

Historically, three multicenter randomized trials investigated the role of CDT against open surgery in ALI: the Rochester, STILE and TOPAS studies. Despite limitations in the heterogeneity of study populations and endpoints, no significant difference in limb salvage or death at 30 days, six months or one year between initial surgery and initial thrombolysis was found [34, 36-39]. The STILE study was the first study to demonstrate a significant outcome based on the duration of ischemia showing that in patients with ischemia $<14$ days the amputation rate for surgery was significantly higher (30\%) compared to thrombolysis with rTPA or urokinase (UK) $(11 \%)$. The trial was prematurely terminated due to the significantly higher incidence of re-thrombosis in the thrombolytic group. A major criticism of the STILE study was that the analysis concerning duration of ischemia (less or more than 14 days) was a post-hoc arbitrary division. However, in the thrombolysis group a higher incidence of stroke, major hemorrhage and distal embolization at 30 days were reported, without any overall difference in limb salvage or death at one year. In a Cochrane review, no evidence could be found either in favor of initial thrombolysis or initial surgery [39]. CDT can be used in combination or as an adjunct to other treatment approaches, after percutaneous thrombus aspiration, mechanical thrombectomy or PTA [40]. Some studies found significantly better patency in patients treated with CDT and angioplasty than in those treated with CDT alone [41].

Percutaneous thrombus aspiration (PAT) via large lumen catheters (6- to 8-French) was the first method of endovascular arterial thrombus extraction, which gained acceptance as a cheap method offering quick recanalization, especially in below-the-knee arteries [29]. Typically, it is utilized as a sole or an adjunctive procedure in patients with thromboembolic complications after endovascular procedures [40]. It is a simple concept, with a stable end-hole catheter being delivered over the guidewire to the site of thrombus burden and a syringe placed at negative pressure being used to induce suction and to aspirate blood and thrombus fragments. It is crucial to use an introducer sheath with a removable hemostatic valve in order to fully retrieve the thrombus burden and to decrease the risk of thromboembolism during the process of thrombus extraction. More atraumatic and flexible catheters, encouraged from stroke approach, allow thrombosuction even from smaller arteries. Surprisingly, the number of studies analyzing the use of aspiration thrombectomy catheters in the peripheral vasculature is low. However, in many interventional institutions, this technique represents the treatment of choice in acute limb ischemia. In a retrospective study Kwok et al. [42] compared thrombolysis with primary aspiration embolectomy. Technical success was achieved approximately in half of the cohort by primary aspiration alone. PAT, if successful as a standalone treatment, obviates the requirement for CDT with a concomitant reduction in the risk of hemorrhagic complications [42]. Factors limiting widespread use are decreased effectiveness in cases of organized thrombus and in large vessels, less favorable results in patients with disseminated atherosclerotic lesions and the risk of inadvertent vessel injury if multiple catheter passages are required unless a long sheath with a removable hemostatic valve is placed close to the occlusion [29].

Mechanical thrombectomy devices can be classified according to the main mechanism of action: mechanical fragmentation, aspiration, rheolytic thrombectomy and combinations thereof. A recent trend is to combine mechanical thrombus removal with thrombolysis. The treatment time decreases compared to thrombolysis alone, and the thrombolytic agent dose can be reduced with a possibly lower bleeding risk. Due to technical limitations, endovascular thrombectomy is not always complete and the treatment of small-diameter vessels is frequently not feasible so that thrombolysis seems to be a reasonable adjunct in such cases [43].

The Rotarex ${ }^{\circledR}$ Device is based on a spiral, connected to the tip of the catheter, rotating at a speed of approximately 40000 revolutions per minute to induce fragmentation of the thrombus. The rapid rotation of the helix generates a permanent vacuum inside the catheter which aspirates the thrombotic material and conveys it to a receptacle. Beneficial primary revascularization rates, especially with respect to elderly patients with multiple medical comorbidities, ranging from $70 \%$ to $96 \%$ have been reported previously [22, 44, 45]. By simultaneously performing aspiration during mechanical fragmentation of the clot, complications such as distal embolization are lower compared to CDT alone [22, 44]. The rapid debulking of the thromboembolism allows partial reperfusion to begin immediately in a controlled selective angiography setting, minimizes ongoing ischemia, and possibly unmasks underlying causative lesions. Concomitant PTA and stenting for the treatment of underlying flow-limiting lesions and stenosis greater than $50 \%$ can possibly lead to a significant improvement of the long-term patency and limb salvage rates. Unfortunately, rotational PMT was not approved for treatment in lesions distal 
to the POP-II segment, and data are missing on rotational PMT in the iliac arteries. Recently, Kronlage et al. [46] compared mechanical thrombectomy to thrombolysis in a retrospective study and found that mechanical thrombectomy was not inferior to local thrombolysis, and that it was associated with a lower complication rate, shorter duration of hospitalization, and lower costs in a 12-moth follow-up.

The Angiojet ${ }^{\circledR}$ Device is based on a hydrodynamic aspiration mechanism. High-speed saline jets are injected through the catheter tip to create a so-called "venturi effect". This results in a lowpressure zone with vacuum effect to simultaneously lyse and aspirate the thrombus [19]. The minimum diameter of treated vessels is $1.5 \mathrm{~mm}$, a fact that allows effective treatment to the level of the crural arteries. The recommended maximum working time is limited to $300 \mathrm{~s}$ while working in the blood stream and $600 \mathrm{~s}$ within the thrombus due to hemolysis that occurs during the procedure [29]. There are prospective and retrospective studies analyzing rheolytic thrombectomy with and without pharmacologic thrombolysis, with an efficacy up to $90 \%$ for recanalization [47, 48]. Potential complications are vessel injury and acute closure, distal embolization and stent graft collapse during thrombectomy, such as acute renal insufficiency and acute pancreatitis due to hemolysis [29].

Isolated Pharmaco-Mechanical Thrombolysis-Thrombectomy System (IPMT) by Trellis device aims to isolate the thrombus between two balloons followed by site-specific thrombolysis. The lysed thrombus is then aspirated while the inflated distal balloon prevents distal embolization. The total procedure time, systemic thrombolytic exposure and therefore bleeding complications are reduced when compared to CDT. Unfortunately, there are no relevant clinical data [49].

Ultrasound-based devices utilize high-frequency low-power ultrasound to enable the delivery of therapeutic agents in the peripheral vasculature. They consist of a reusable control system that powers the unit and a single-use infusion catheter system. The latter includes a drug delivery catheter (DDC) containing an ultrasound core (USC). It separates clot fibrin for better drug delivery into the clot without fragmentation of emboli and the principle of acoustic streaming hastens the penetration of the lytic agent into the clot. Ultrasound-based devices may provide a safe, effective, and time-saving treatment option. A few studies with small population sizes confirm high technical success rates of up to $100 \%$. However, clinical experience is limited and randomized studies are still missing [50]. These studies presume more rapid recanalization and a higher rate of early complete thrombus resolution by ultrasound-based devices compared to conventional lysis, thus reducing the hospitalization time [51].

\section{Surgical Revascularization}

Surgical revascularization is the treatment of choice in category lla or IIb ALI with symptoms > 14 days, in the case of contraindications to thrombolysis or an endovascular approach, or in suprainguinal occlusion $[1,8,40]$.
Fogarty balloon catheter embolectomy was developed by Fogarty in 1963 [52]. After the advent of this technique, embolectomy and thrombectomy became easy and secure by approaching from the femoral artery. The procedure involves surgical exposure of the artery, usually at the common femoral or popliteal arteries. A horizontal arteriotomy is usually performed in embolic ALI, stenosis in subsequent closure can thus be minimized. In suspected thrombotic ALI on the other hand, a vertical arteriotomy is preferred. In that case, if subsequent bypass is needed, the same arteriotomy can be used for graft anastomosis. If no bypass is required, the arteriotomy should be closed using a vein patch to minimize potential stenosis [8]. Factors related to a successful outcome of the treatment of arterial embolism are an intact arterial intima, non-adherence of the thromboembolism to the intima, and a patent run-off vessel prior to embolization [4]. The catheter is introduced and pushed proximally beyond the thrombus and extracted after inflation of the balloon. This process is performed several times until all visible clots are removed and back bleeding is achieved [8]. After finishing the procedure, a completion angiogram in the operating room is recommended to exclude residual embolus and distal embolization and to secure patency of distal arteries, which allows immediate re-intervention [20].

Bypass procedures can be necessary in patients with ALI caused by thrombosis of underlying atherosclerotic disease. Patients with atherosclerotic native arteries tend to suffer from rethrombosis, if balloon embolectomy is performed.

As mentioned above similar outcomes have been reported when comparing open surgical treatment with CDT with respect to amputation-free survival and overall mortality [34, 36-38].

While higher bleeding complications have been reported in thrombolysis, surgery showed increased morbidity related to cardiopulmonary complications, blood loss, wound infections and fasciotomy [53].

\section{Conclusion}

Acute limb ischemia is an interdisciplinary emergency, with variable presentation that may be life-threatening and lead to limb loss. Immediate diagnosis and treatment are critical to prevent irreversible damage. An endovascular approach should be considered in ALI categories Ila and IIb $<14$ days, on a case-based decision, with special regard to elderly multimorbid patients with a high perioperative risk. In this setting, the structural, personal and technical conditions of each department must be considered.

\section{Conflict of Interest}

Thorsten Alexander Bley: Employment: Chairman Department of Diagnostic and Interventional Radiology, Julius-Maximilians-University of Würzburg, Scientific research support, Deutsche Forschungsgemeinschaft (DFG), Siemens Healthineers; Consulting: GSK, HeartFlow, MSD, Roche/Chugai; Honoraria: Bayer, Bracco, Guerbet, HeartFlow, Roche/ Chugai, Siemens.

Ralph Kickuth: Vortragshonorar: Schöchl medical education GmbH, Mattsee, Österreich. 
[1] Norgren L, Hiatt WR, Dormandy JA et al. Inter-Society Consensus for the Management of Peripheral Arterial Disease (TASC II). European Journal of Vascular \& Endovascular Surgery 2007; 33: S1-S75

[2] Byrne RM, Taha AG, Avgerinos E et al. Contemporary outcomes of endovascular interventions for acute limb ischemia. Journal of Vascular Surgery 2014; 59: 988-995

[3] Patel NH, Krishnamurthy VN, Kim S et al. Quality improvement guidelines for percutaneous management of acute lower-extremity ischemia. J Vasc Interv Radiol 2013; 24: 3-15

[4] Fukuda I, Chiyoya M, Taniguchi S et al. Acute limb ischemia: contemporary approach. General Thoracic and Cardiovascular Surgery 2015; 63: 540-548

[5] Gerhard-Herman MD, Gornik HL, Barrett C et al. 2016 AHA/ACC Guideline on the Management of Patients With Lower Extremity Peripheral Artery Disease: A Report of the American College of Cardiology/American Heart Association Task Force on Clinical Practice Guidelines. Circulation 2017; 135: e726-e779

[6] Hardman R, Jazaeri O, Yi J et al. Overview of Classification Systems in Peripheral Artery Disease. Semin intervent Radiol 2014; 31: 378-388

[7] Rutherford RB, Baker JD, Ernst C et al. Recommended standards for reports dealing with lower extremity ischemia: revised version. Journal of Vascular Surgery 1997; 26: 517-538

[8] Gunawansa N. Atraumatic Acute Limb Ischemia: Clinical Presentation, Classification, Assessment and Management- A Review. J Vasc Surg Med 2017; 1: 10-15

[9] Kashyap VS, Gilani R, Bena JF et al. Endovascular therapy for acute limb ischemia. Journal of Vascular Surgery 2011; 53: 340-346

[10] Taha AG, Byrne RM, Avgerinos ED et al. Comparative effectiveness of endovascular versus surgical revascularization for acute lower extremity ischemia. Journal of Vascular Surgery 2015; 61: 147-154

[11] Vaidya V, Gangan N, Comerota A et al. Cost-Effectiveness Analysis of Initial Treatment Strategies for Nonembolic Acute Limb Ischemia Using Real-Word Data. Annals of Vascular Surgery 2017; 39: 276-283

[12] Hage A, McDevitt J, Chick J et al. Acute Limb Ischemia Therapies: When and How to Treat Endovascularly. Semin intervent Radiol 2019; 35: $453-$ 460

[13] Weiss CR, Azene EM, Majdalany BS et al. ACR Appropriateness Criteria Sudden Onset of Cold, Painful Leg. Journal of the American College of Radiology 2017; 14: 307-313

[14] Andreucci M, Faga T, Serra R et al. Update on the renal toxicity of iodinated contrast drugs used in clinical medicine. Drug Healthc Patient Saf 2017; 9: 25-37

[15] Berg F, Bangard C, Bovenschulte $H$ et al. Hybrid contrast-enhanced MR angiography of pelvic and lower extremity vasculature at 3.0T: Initial experience. European Journal of Radiology 2009; 70: 170-176

[16] Hadizadeh DR, Gieseke J, Lohmaier SH et al. Peripheral MR Angiography with Blood Pool Contrast Agent: Prospective Intraindividual Comparative Study of High-Spatial-Resolution Steady-State MR Angiography versus Standard-Resolution First-Pass MR Angiography and DSA. Radiology 2008; 249: 701-711

[17] Thomas C, Korn A, Ketelsen D et al. Automatic Lumen Segmentation in Calcified Plaques: Dual-Energy CT Versus Standard Reconstructions in Comparison with Digital Subtraction Angiography. American Journal of Roentgenology 2010; 194: 1590-1595

[18] Blaisdell FW, Steele M, Allen RE. Management of acute lower extremity arterial ischemia due to embolism and thrombosis. Surgery 1978; 84 : 822-834

[19] Valle JA, Waldo SW. Current Endovascular Management of Acute Limb Ischemia. Interventional Cardiology Clinics 2017; 6: 189-196
[20] Karnabatidis D, Spiliopoulos S, Tsetis D et al. Quality Improvement Guidelines for Percutaneous Catheter-Directed Intra-Arterial Thrombolysis and Mechanical Thrombectomy for Acute Lower-Limb Ischemia. Cardiovasc Intervent Radiol 2011; 34: 1123-1136

[21] Kessel DO, Berridge DC, Robertson I. Infusion techniques for peripheral arterial thrombolysis. Cochrane Database of Systematic Reviews 2004; 78: $988-1020$

[22] Lichtenberg M. Percutaneous mechanical thrombectomy by means of rotational thrombectomy. Current study situation. Med Klin (Munich) 2010; 105: 705-710

[23] Wang JC, Kim AH, Kashyap VS. Open surgical or endovascular revascularization for acute limb ischemia. Journal of Vascular Surgery 2016; 63: 270-278

[24] Diffin DC, Kandarpa K. Assessment of peripheral intraarterial thrombolysis versus surgical revascularization in acute lower-limb ischemia: a review of limb-salvage and mortality statistics. J Vasc Interv Radiol 1996; 7: $57-63$

[25] Lichtenberg M, Kaeunicke M, Hailer B. Percutaneous mechanical thrombectomy for treatment of acute femoropopliteal bypass occlusion. VHRM 2012; 8: 283-289

[26] Korabathina R, Weintraub AR, Price LL et al. Twenty-Year Analysis of Trends in the Incidence and In-Hospital Mortality for Lower-Extremity Arterial Thromboembolism. Circulation 2013; 128: 115-121

[27] Rajan DK, Patel NH, Valji K et al. Quality improvement guidelines for percutaneous management of acute limb ischemia. J Vasc Interv Radiol 2005; 16: 585-595

[28] Aboyans V, Ricco JB, Bartelink MLEL et al. 2017 ESC Guidelines on the Diagnosis and Treatment of Peripheral Arterial Diseases, in collaboration with the European Society for Vascular Surgery (ESVS): Document covering atherosclerotic disease of extracranial carotid and vertebral, mesenteric, renal, upper and lower extremity arteries Endorsed by: the European Stroke Organization (ESO)The Task Force for the Diagnosis and Treatment of Peripheral Arterial Diseases of the European Society of Cardiology (ESC) and of the European Society for Vascular Surgery (ESVS). Eur Heart J 2018; 39: 763-816

[29] Lukasiewicz A. Treatment of acute lower limb ischaemia. Vasa 2016; 45 : 213-221

[30] Grip O, Kuoppala M, Acosta S et al. Outcome and complications after intra-arterial thrombolysis for lower limb ischaemia with or without continuous heparin infusion. Br J Surg 2014; 101: 1105-1112

[31] Schrijver AM, van Leersum M, Fioole B et al. Dutch Randomized Trial Comparing Standard Catheter-Directed Thrombolysis and UltrasoundAccelerated Thrombolysis for Arterial Thromboembolic Infrainguinal Disease (DUET). J Endovasc Ther 2015; 22: 87-95

[32] Schrijver AM, Reijnen MMPJ, van Oostayen JA et al. Dutch randomized trial comparing standard catheter-directed thrombolysis versus ultrasound-accelerated thrombolysis for thromboembolic infrainguinal disease (DUET): design and rationale. Trials 2011; 12: 20

[33] Elizabeth LG, Colvard B, Ho VT et al. Real-World Outcomes of EKOS Ultrasound-Enhanced Catheter-Directed Thrombolysis for Acute Limb Ischemia. Journal of Vascular Surgery 2019; 69: e170-e171

[34] Han SM, Weaver FA, Comerota AJ et al. Efficacy and safety of alfimeprase in patients with acute peripheral arterial occlusion (PAO). Journal of Vascular Surgery 2010; 51: 600-609

[35] Johner F, Clemens R, Husmann M et al. Long-term follow-up after endovascular revascularisation for acute limb ischaemia-a retrospective single-centre cohort. Vasa 2016; 45: 247-252

[36] Ouriel K, Veith F], Sasahara AA. Thrombolysis or peripheral arterial surgery: phase I results. TOPAS Investigators. Journal of Vascular Surgery 1996; 23: 64-73; discussion74-75

[37] Ouriel K, Veith F], Sasahara AA. A comparison of recombinant urokinase with vascular surgery as initial treatment for acute arterial occlusion of 
the legs. Thrombolysis or Peripheral Arterial Surgery (TOPAS) Investigators. N Engl J Med 1998; 338: 1105-1111

[38] The STILE Investigators. Results of a prospective randomized trial evaluating surgery versus thrombolysis for ischemia of the lower extremity. The STILE trial. Ann Surg 1994; 220: 251-268

[39] Berridge DC, Kessel DO, Robertson I. Surgery versus thrombolysis for initial management of acute limb ischaemia. Cochrane Database of Systematic Reviews 2013. doi:10.1002/14651858.CD002784.pub2

[40] Zehnder T, Birrer M, Do DD et al. Percutaneous Catheter Thrombus Aspiration for Acute or Subacute Arterial Occlusion of the Legs: How Much Thrombolysis is Needed? European Journal of Vascular \& Endovascular Surgery 2000; 20: 41-46

[41] Urbak L, de la Motte L, Rørdam P et al. Catheter-Directed Thrombolysis in the Treatment of Acute Ischemia in Lower Extremities Is Safe and Effective, Especially with Concomitant Endovascular Treatment. Annals of Vascular Diseases 2017; 10: 125-131

[42] Kwok CHR, Fleming S, Chan KKC et al. Aspiration Thrombectomy versus Conventional Catheter-Directed Thrombolysis as First-Line Treatment for Noniatrogenic Acute Lower Limb Ischemia. J Vasc Interv Radiol 2018; 29: $607-613$

[43] Leung DA, Blitz LR, Nelson T et al. Rheolytic Pharmacomechanical Thrombectomy for the Management of Acute Limb Ischemia: Results From the PEARL Registry. J Endovasc Ther 2015; 22: 546-557

[44] Wissgott C, Kamusella P, Richter A et al. Mechanical rotational thrombectomy for treatment thrombolysis in acute and subacute occlusion of femoropopliteal arteries: retrospective analysis of the results from 1999 to 2005. Rofo 2008; 180: 325-331
[45] Stanek F, Ouhrabkova R, Prochazka D. Percutaneous mechanical thrombectomy in the treatment of acute and subacute occlusions of the peripheral arteries and bypasses. Vasa 2016; 45: 49-56

[46] Kronlage M, Printz I, Vogel B et al. A comparative study on endovascular treatment of (sub)acute critical limb ischemia: mechanical thrombectomy vs thrombolysis. DDDT 2017; 11: 1233-1241

[47] Wagner HJ, Müller-Hülsbeck S, Pitton MB et al. Rapid thrombectomy with a hydrodynamic catheter: results from a prospective, multicenter trial. Radiology 1997; 205: 675-681

[48] Allie DE, Hebert C], Lirtzman MD et al. Novel simultaneous combination chemical thrombolysis/rheolytic thrombectomy therapy for acute critical limb ischemia: The power-pulse spray technique. Catheterization and Cardiovascular Interventions 2004; 63: 512-522

[49] Gupta R, Hennebry TA. Percutaneous isolated pharmaco-mechanical thrombolysis-thrombectomy system for the management of acute arterial limb ischemia: 30-day results from a single-center experience. Catheter Cardiovasc Interv 2012; 80: 636-643

[50] Wissgott C, Richter A, Kamusella P et al. Treatment of Critical Limb Ischemia Using Ultrasound- Enhanced Thrombolysis (PARES Trial): Final Results. J Endovasc Ther 2007; 14: 438-443

[51] Acar RD, Sahin M, Kirma C. One of the most urgent vascular circumstances: Acute limb ischemia. SAGE Open Medicine 2013. doi:10.1177| 2050312113516110

[52] Fogarty TJ, Cranley J], Krause RJ et al. A method for extraction of arterial emboli and thrombi. Surg Gynecol Obstet 1963; 116: 241-244

[53] Wang JC, Kim AH, Kashyap VS. Open surgical or endovascular revascularization for acute limb ischemia. Journal of Vascular Surgery 2016; 63: 270-278 